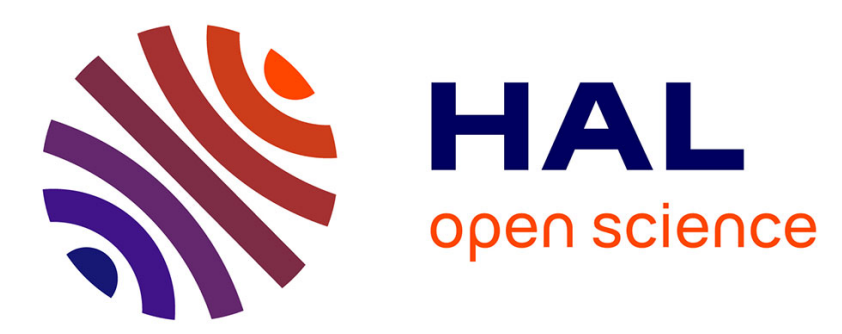

\title{
Les supra-commandements comme solution à la crise militaire du IIIe siècle de l'Empire romain sous Philippe l'Arabe et Gallien
}

Hermann Amon

\section{- To cite this version:}

Hermann Amon. Les supra-commandements comme solution à la crise militaire du IIIe siècle de l'Empire romain sous Philippe l'Arabe et Gallien. Archimède : archéologie et histoire ancienne, 2016, 3, pp.206-217. hal-01587268

\section{HAL Id: hal-01587268 \\ https://hal.science/hal-01587268}

Submitted on 13 Sep 2017

HAL is a multi-disciplinary open access archive for the deposit and dissemination of scientific research documents, whether they are published or not. The documents may come from teaching and research institutions in France or abroad, or from public or private research centers.
L'archive ouverte pluridisciplinaire HAL, est destinée au dépôt et à la diffusion de documents scientifiques de niveau recherche, publiés ou non, émanant des établissements d'enseignement et de recherche français ou étrangers, des laboratoires publics ou privés. 


\section{DOSSIER THÉMATIQUE : DES FOSSÉS ET DES REMPARTS. ENCEINTES ET SITES FORTIFIÉS DU RHIN SUPÉRIEUR ENTRE PROTOHISTOIRE ET MOYEN ÂGE}

\section{Olivier BUCHSENSCHUTZ}

Avant-propos. Des enceintes en terre anhistoriques à Google Earth

8 Lizzie SCHOLTUS

Histoire de la recherche dans le bassin de Saint-Dié-des-Vosges

20 Maxime WALTER

Les sites de hauteur du massif vosgien. Actualisation des données et modalités d'implantation

37 Jean-Jacques SCHWIEN

Chateaux et enceintes des Vosges du Nord. Topographie et longue durée

49 Anne-Marie ADAM

La palissade dans tous ses états : I'enclos du Britzgyberg (Illfurth, Haut-Rhin) et autres aménagements palissadés dans les habitats du premier âge du Fer

60 Clément FÉLIU

L'enceinte inférieure du Frankenbourg (67) et les remparts à poteaux frontaux de la fin de l'âge du Fer dans l'espace du Rhin supérieur. Pour une révision de la typologie des Pfostenschlitzmauern

74 Jacky $\mathrm{KOCH}$ et Thomas FISCHBACH

Enceintes de hauteur en pierres et formes « primitives » de châteaux ? L'exemple du Bernstein

87 Adrien VUILLEMIN

Les enceintes urbaines en moyenne Alsace (1200-1850)

102 Jean-François PININGRE

Les enceintes de l'âge du Bronze et du premier âge du Fer en Franche-Comté. Un bilan des recherches

124 Clément FÉLIU et Jean-Jacques SCHWIEN

Conclusion. Nouvelles perspectives sur les enceintes du Rhin supérieur

\section{ACTUALITÉ DE LA RECHERCHE : ARCHÉOLOGIE DES RÉSEAUX}

\section{Claire CAMBERLEIN}

Les réseaux en archéologie : approche historiographique et interdisciplinaire

135 Thomas HUTIN

Lieux d'échanges et espaces publics en Gaule à La Tène finale

150 Streeve GENTNER

Économie du fer et voies de communication, de l'abattage du minerai à la distribution

du métal : I'exemple du nord de la Forêt-Noire au Ve siècle av. J.-C.

169 Loup BERNARD et Rémy WASSONG

Du Danemark au Fossé rhénan. Un siècle d'analyse des voies de communications protohistoriques : évolution des méthodes et mise en commun des données

184 Steeve GENTNER et Rémy WASSONG

Conclusion. L'archéologie des réseaux : une thématique aux multiples facettes

\section{VARIA}

187 Fábio VERGARA CERQUEIRA

To march in phalanx, to jump with weights, to tread the grapes, to knead the bread. What is the aulos for?

\section{Hermann AMON}

Les supra-commandements comme solution à la crise militaire du III siècle de l'Empire romain sous Philippe l'Arabe et Gallien

218 Martina BONO

Il processo di Cremuzio Cordo in Dio LVII, 24, 2-4

\section{LA CHRONIQUE D'ARCHIMÈDE}

228 Frédéric COLIN (éd.)

La Chronique d'Archimède. Bilan des activités scientifiques 2015-2016 de I'unité mixte de recherche 7044 


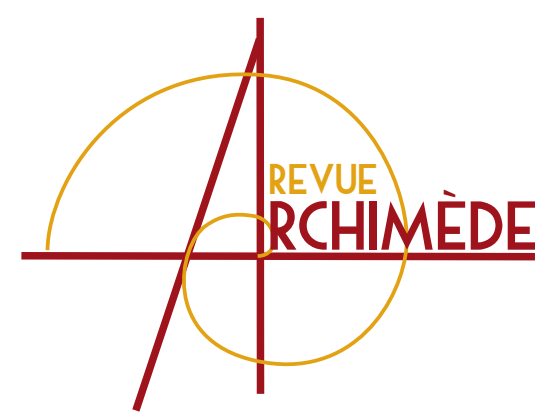

\title{
LES SUPRA-COMMANDEMENTS COMME SOLUTION À LA CRISE MILITAIRE DU IIII SIÈCLE DE L'EMPIRE ROMAIN SOUS PHILIPPE L'ARABE ET GALLIEN
}

\author{
Hermann AMON \\ Chercheur Associé au Réseau pour \\ l'Étude de l'Antiquité tardive \\ Université d'Ottawa \\ hamon@uottawa.ca
}

\section{RÉSUMÉ}

Au III $^{\mathrm{e}}$ siècle, I'intensification et la simultanéité des attaques germaniques et perses aux frontières de l'Empire romain conduit certains empereurs à mettre en place des supra-commandements dans le cadre de leur stratégie de lutte contre les invasions. Ces supracommandements étaient des structures de coordination placées à la tête de plusieurs provinces dont la direction était confiée à un seul individu. Ils avaient pour objectif de faciliter une mutualisation des efforts militaires au sein des principales régions menacées d'attaques. L'objectif de cet article est d'analyser leur impact dans le refoulement des invasions et la réduction des coups d'État sous les règnes de Philippe I'Arabe et Gallien. Les supracommandements de Priscus, de Valérien le Jeune et d'Odenath de Palmyre serviront de cadre de référence.
During the third century, intensification and simultaneity of germanic and persian attacks at the Roman Empire's borders pushed some emperors to put in place supraprovincial commands as part of their strategy against invasions. These supra-provincial commands were coordination structures with jurisdiction over many provinces, and were led by a single individual. Their objective was to concert military efforts within main areas subject to possible attacks. This paper examines the impact of these structures on repelling invasions and reducing political coups under the reigns of Philip the Arab and Gallienus. The supra-provincial commands of Priscus, Valerian the Younger and Odenath of Palmyra will be used as Supra-provincial commands, references.
KEYWORDS third century crisis, invasions, political coup. 


\section{INTRODUCTION}

L'urgente nécessité d'assurer une coordination locale des efforts de protection des régions menacées d'attaques par les ennemis extérieurs de l'empire, conduit certains empereurs à mettre en place à partir de 235 des supra-commandements. Il faut entendre par supra-commandements, des structures de coordination placées à la tête de plusieurs provinces dont la direction était confiée à un seul individu. Leur spécificité se situe non seulement dans I'unique autorité de supervision sur les gouverneurs de provinces accordée à son détenteur mais également dans leur fonction essentiellement militaire. En outre, ils résolvaient le dilemme qui se posait à certains empereurs du $\mathrm{II}^{\mathrm{e}}$ siècle, qui était celui de leur nécessaire mais impossible présence sur plusieurs foyers de tension.

Cette réflexion sur les supra-commandements est pertinente à plus d'un titre puisqu'elle améliore notre connaissance des structures administratives qui résultèrent des assauts germaniques et perses aux frontières de l'empire au III siècle. Ils ont d'ailleurs fait l'objet de plusieurs articles qui se sont le plus souvent intéressés aux personnages qui les ont exercés et aux pouvoirs qu'ils détenaient. Il faut mentionner à cet égard l'article de Ronald Syme qui décrit les pouvoirs accordés à Avidius Cassius, commandant des provinces orientales sous le règne de Marc Aurèle [1]. Les articles de Simon Swain [2] et de David Potter [3] discutent quant à eux le contenu véritable des pouvoirs d'Odenath sur la base de deux inscriptions honorifiques trouvées à Palmyre [4]. La contribution de Frederik Vervaet [5] va plus loin que les trois précédentes. Elle part d'une présentation générale des supra-commandements dans l'empire pour analyser

[1] SYME 1984-1985, p. 217-222.

[2] SWAin 1993, p. 157-164.

[3] POTTER 1996, p. 271-285.

[4] CIS II 3946 ; CIS II 3971.

[5] VeRVAET 2007, p. 125-140. la nature de ceux détenus par Pline le Jeune en Bithynie, Avidius Cassius et Iulius Priscus en Orient. La nouveauté de notre présente analyse se situe dans la mise en perspective des supra-commandements avec la crise militaire aux frontières de l'empire au III siècle. L'objectif essentiel de cet article est d'évaluer leur impact dans la résolution de cette crise militaire et politique sous les règnes de Philippe I'Arabe et Gallien et ce, à travers deux indicateurs que sont la diminution des invasions et la réduction des Coups d'État. Les supra-commandements de Priscus, de Valérien le Jeune et d'Odenath serviront de cadre de référence à cet effet. Nous présenterons, pour chacun d'eux, le contexte de leur mise en place, dans la mesure du possible leur contenu administratif ou politique et leur impact sur la diminution des attaques aux frontières et des coups d'État.

\section{PHILIPPE L'ARABE ET LE SUPRA-COMMANDEMENT ORIENTAL}

Philippe l'Arabe fut le premier empereur du III ${ }^{\mathrm{e}}$ siècle à mettre en place des supra-commandements. En effet, il prit le pouvoir en mars 244 à l'issue d'un coup d'État mené contre le jeune empereur Gordien III [6]. Après son acclamation par les soldats, il négocia un accord de paix dans en vue de mettre fin à la guerre en cours avec les Perses [7]. Ce compromis politique, qui constitue l'épilogue du second conflit entre Romains et Perses, comprenait le versement d'un tribut à ces derniers, en contrepartie d'un retour sur le plan territorial aux frontières de 238 [8]. Ainsi, les provinces d'Osrhoène et de Mésopotamie reconquises sous le commandement de

[6] LORIOT 1975b, p. 789.

[7] Zosime, I, 19, 1 ; Sapor dans ses Res Gestae Divi Saporis (RGDS) indique qu'il s'agissait d'un tribut annuel de 500000 aurei. Il prétend même que Philippe devint dès lors son «tributaire», RGDS, I, 9. Voir également PEKÀRY 1961 , p. 277-278. 
Timésithée furent préservées [9]. Dans le même temps, les Romains devaient renoncer au soutien diplomatique et militaire qu'ils apportaient au royaume de Grande Arménie. Ils laissaient ainsi le champ libre aux Perses contre Chosroês le Grand [10]. Il faut souligner que cette dernière clause n'aurait pas été incluse dans l'accord officiel. D'ailleurs Sapor dans ses Res Gestae fait état de la volte-face de Philippe au sujet de I'Arménie [11]. S'appuyant certainement sur ce commentaire du souverain perse, Zonaras indique que Philippe après s'être engagé à céder l'Arménie et la Mésopotamie aux Iraniens, les aurait reconquises face à la pression de l'opinion publique romaine [12]. Cette version des faits n'est pas rapportée pas d'autres sources et nous n'avons aucune trace d'un retour à l'état de guerre entre les deux parties. Il est probable que ce commentaire de Sapor soit l'expression de son exaspération face à la lenteur du désengagement romain d'Arménie, lourd de conséquence pour l'équilibre des forces dans la région [13].

En tout état de cause, cet accord de paix fut célébré avec faste par les deux parties, Sapor se considérant comme le tributaire de Philippe [14] et Philippe revêtant sans complexe les titres de PARTHICVS MAXIMVS et de PERSICVS MAXIMVS [15]. Cette revendication de la victoire par les deux souverains confirmait sans l'ombre d'un doute la permanence de la tension aux frontières des deux empires. C'est donc conscient de cette stabilité précaire dans cette région hautement stratégique que Philippe décida d'y créer un supra-commandement, qu'il confia à son frère Priscus. C'est Zosime qui fait mention de sa mise en place :

«Il jugea nécessaire de confier les commandements les plus élevés à ceux qui lui étaient les plus proches, mit son frère Priscus à la tête des légions de Syrie et confia à son beau-frère Sévérien les forces stationnées en Mésie et en Macédoine » [16].

[8] LORIOT 1975a, p. 774.

[9] La direction de la province de Mésopotamie fut plus tard confiée à Priscus.

[10] Christol 2006, p. 100 ; Chaumont 1969, p. 41-46.

[11] MARICQ 1958, p. 308

[12] Zonaras, XII, 19.

[13] Chaumont 1969, p. 44-47.

[14] MARICQ 1958, p. 308.

[15] CIL III 4634 ; CIL III 10619 ; ILS 597.

[16] Zosime, I, 19, 2.

[17] Nous savons peu de choses sur Sévérien. Il serait le frère de Marcia Octacilia Severa, I'épouse de Philippe. Issu de la célèbre gens Otacilia, il aurait probablement gravi les échelons de la carrière sénatoriale avant d'obtenir le gouvernement de la province impériale de Mésie supérieure puis le second grand commandement qui couvrait les provinces danubiennes. Voir à ce propos KöRnER 2002, p. 63.

[18] $P I R^{2}$ IV 3, p. 254, n488. CIL VI 1638 ; KÖRNER 2002, p.
Les deux personnages en question sont Caius Iulius Priscus et Sévérien [17]. Caius Iulius Priscus était le frère aîné de l'empereur Philippe [18]. Nous savons qu'il était membre de l'ordre équestre. L'ascension de Timésithée, sous le règne de Gordien III, lui permit d'accéder aux hautes fonctions de la carrière équestre [19]. Il occupa la préfecture du prétoire à deux reprises ; d'abord comme collègue de Timésithée entre 242 et 243 [20] sous le règne de Gordien III et durant les dernières années de règne de Philippe [21]. Après la prise de pouvoir de Philippe en mars 244, il reçut une large autorité sur les provinces orientales. Quel type d'autorité exerçait Priscus sur le supra-commandement oriental ?

Selon les documents d'archive (cf. infra): archives provenant du Moyen Euphrate, Priscus était, au mois d'août 245, préfet de Mésopotamie tout en exerçant le «gouvernement consulaire» de la Syrie [22]. Une dédicace latine de Timgad datant de 247-249 vient compléter ses titres en mentionnant celui de Rector Orientis [23].

Nous serions en présence d'une véritable entorse à la pratique administrative si Priscus assurait le commandement direct des deux provinces. En effet, demeurémembre del'ordre équestre, ce dernier ne pouvait prétendre au commandement d'une province sénatoriale comme la Syrie-Coelé qui était administrativement placée sous l'autorité d'un légat d'Auguste propréteur de rang consulaire [24]. En réalité, Priscus exerçait déjà le commandement de la province équestre de Mésopotamie avant le 28 août 245 [25]. Philippe lui confia la direction de cette province stratégique après sa reconquête à I'issue du conflit avec les Perses [26]. En plus de la Mésopotamie, il reçut le commandement intérimaire de la province consulaire de Syrie-Coelé de la période allant de décembre 244 à août 245 [27]. L'intérim de Priscus est confirmé par les mêmes documents d'archives

54-63 ; Pflaum 1960, p. 831-839 ; Feissel \& Gascou 1995, p. 65-119 ; HOWE 1942, p.106-111; NASTI 1993, p. 365-380 ; NASTI 1997, p. 281-290.

[19] De BLoIS 1978-1979, p. 11-12.

[20] Feissel \& GASCOU 1995, p. 81.

[21] CIL III 14149 ; ILS 9005 ; KöRNER 2002, p. 55-57.

[22] FeIsSel \& GASCOU 1995, p. 65-119; KÖRNER 2002, p. 57.

[23] Voir CIL III 14149 ; ILS 9005. C. IUl(io) $\operatorname{Pr}[i] s c o$, $v\{$ iro) em(inentissimo), fratr[i] et patru[o] d(ominorum) $n$ [ostrorum) Philipporum Aug\{ustorum) et praef\{ecto) praet(orio) rectori(q]ue) Orientis, Trebonius Sossianus, p\{rimi)p(ilaris), domo col(onia) Hel(iopoli), devotus numini majestati[q(ue)]eorum.

[24] LefebVRe 2011, p. 114-115.

[25] Feissel \& GASCOU 1995 p. 81.

[26] Trout 1989, p. 228-229.

[27] Feissel \& Gascou 1995, p. 81. Priscus occupa le commandement intérimaire depuis huit mois avant la date d'août 245 ; BenOISt 2000, p. 322-323. 
(cf. infra) : archive du Moyen-Euphrate puisqu'on y fait mention d'un certain Marcellus de rang perfectissime qui exerçait le même gouvernement intérimaire de la province de Syrie-Coelé [28]. Ce dernier aurait occupé cette fonction intérimaire après le départ de Priscus, c'est-à-dire à partir d'août 245 [29]. Pendant cette période, Priscus combinait le commandement direct des deux provinces (la province équestre de Mésopotamie et la province sénatoriale de Syrie) [30]. C'est probablement après cet intérim en Syrie qu'il fut honoré par Philippe du titre de Rector Orientis. Ce titre consacrait son supra-commandement oriental et lui conférait une autorité de supervision sur l'ensemble des provinces orientales en plus de son commandement direct sur la Mésopotamie [31]. Christian Körner suggère que ce titre de Rector Orientis accordait à Priscus un commandement direct sur l'ensemble des provinces orientales [32]. Nous ne sommes pas de cet avis puisqu'aucune évidence littéraire ou épigraphique ne permet de soutenir cette assertion. Nous pensons en revanche que ce titre n'avait probablement pas de contenu spécifique en matière de droit public mais sur le plan politique et sur la coordination des activités militaires, il octroyait à son détenteur une préséance sur les autres gouverneurs de provinces. Celui-ci était I'homme de confiance de l'empereur dans la région et comme tel avait une autorité de supervision sur l'ensemble des forces militaires positionnées en Orient [33]. Le supracommandement de Priscus couvrait certainement les Syrie, la Mésopotamie, I'Osroène, la Palestine et I'Arabie [34]. Quelles étaient les responsabilités de Priscus dans le cadre de son supra-commandement?

On dispose malheureusement de peu d'informations sur le détail des attributions de Priscus. Nous savons grâce aux documents d'archives mentionnés plus haut qu'il administrait la justice à Antioche [35]. Il s'agit cependant d'une attribution liée à son intérim de gouverneur de Syrie-Coelé. Ses responsabilités sur le supra-commandement oriental couvraient assurément tout ce qui

[28] Feissel \& Gascou 1995, p. 88.

[29] Ibid., p. 92-93.

[30] ECK 1992, p. 201 ; Badel \& Beranger 1998, p. 190.

[31] Vervaet 2007, p. 136-137; Christol 2006, p. 104 ; BENOIST 2000, p. 322-323.

[32] KÖRNER 2002, p. 59.

[33] MiLLAR 1993, p. 156.

[34] POTTER 2004, p. 239.

[35] FeISSEL \& GASCOU 1995, p. 72.

[36] Zosime, I, 20, 2.

[37] PotTer 1990, p. 246 ; PeKÁry 1961, p. 279.

[38] LORIOT 1975a, p. 681. était du domaine militaire dont les finances publiques. En d'autres termes, il avait un droit de regard sur le processus de collecte des impôts dans la région. En effet, selon Zosime, Priscus se montra très rigide dans le recouvrement des impôts auprès des provinciaux [36]. Les finances publiques étaient en piteux état tout au long du règne de Philippe à cause notamment du tribut versé aux Perses à l'issue du conflit oriental et des festivités organisées à I'occasion du millénaire de Rome [37]. La crise militaire aux frontières exigeait une mobilisation permanente de ressources financières pour y faire face. L'inflexibilité fiscale de Priscus s'inscrivait donc dans cette recherche de ressources financières additionnelles. Il est probable qu'il ait demandé aux gouverneurs de provinces de la région d'user de techniques identiques à celles de Maximin le Thrace en Afrique proconsulaire [38]. Par voie de conséquence, il se serait donc mis à dos les dignitaires locaux qui exprimèrent leur désaccord en soutenant Jotapien. La création des supra-commandements s'inscrit dans la perspective de faciliter une coordination locale des opérations militaires. En conséquence, Priscus avait principalement autorité pour définir et mettre en œuvre la politique de sécurisation de la frontière orientale [39].

Analysons maintenant l'impact de ce supra-commandement oriental sur les coups d'État et sur les attaques à la frontière sous le règne de Philippe. En ce qui concerne les coups d'État, il faut souligner que la pression fiscale exercée par Priscus fut à I'origine du coup d'État de Jotapien [40].

«La situation était très troublée dans ce même temps : I'Orient, accablé par les levées des impôts et par le fait que Priscus qui avait la charge de gouverner les provinces dans cette région était insupportable à chacun, et enclin par conséquent à fomenter des troubles, éleva au pouvoir suprême Jotapien » [41].

La population civile et les dignitaires locaux, excédés par la brutalité de la collecte des impôts, se rebellèrent contre l'autorité de Priscus et proclamèrent un certain Jotapien comme empereur [42]. La région précise où

[39] Un pouvoir comparable sur l'Orient tout entier est prêté par Zosime, I, 60, 1, à Marcellinus, qui fut sous l'empereur Aurélien, en 272, à la fois préfet de Mésopotamie et gouverneur de I'Orient.

[40] Ce coup d'État pourrait s'être déroulé en Cappadoce ou en Syrie. Voir à ce propos: Aurelius Victor, Liber de Caesaribus, XXIX, 2 ; Polemius Silvius Laterculus (MommSEn, Chronica minora, I, p. 521, I. 38 ; cf. Mommsen, Gesammelte Schriften, 7, p. 644); Zosime I, 20, 2 et I, 21, 2. Le coup d'État de Jotapien est aussi mentionné dans les Oracles Sibyllins, 13, 89-102. Voir, à ce propos, POTTER 1990, p. 268273 ; Kettenhofen 1982, p. 84 ; Kienast 1990, p. 201.

[41] Zosime, I, 20, 2.

[42] Aurelius Victor, Liber de Caesaribus, XXIX, 2 ; KöRNER 2002, p. 280. 
se déroula cette proclamation ne nous est pas clairement indiquée. Toutefois, il pourrait s'agir soit de la Syrie soit de la Cappadoce. Probablement parti de Cappadoce entre la fin de 248 et le début de 249, ce coup d'État se serait étendu à d'autres régions orientales dont la Syrie et la Mésopotamie [43]. Concernant l'auteur de ce mouvement nous ne savons presque rien. Le témoignage des auteurs anciens fournit très peu d'informations sur le personnage. Zosime, qui mentionne ce coup d'État, ne fournit aucun renseignement sur l'identité de Jotapien ou encore sur son statut social. Il indique seulement son nom. Dans une brève allusion, Aurelius Victor souligne que Jotapien se faisait passer pour un descendant d'Alexandre le Grand [44]. Se fondant sur cette affirmation, Ronald Syme indique que le nom Iotapianus est semblable à ceux des reines Iotape I et II de la dynastie royale des Commagènes, reines qui prétendaient descendre d'Alexandre le Grand. Par conséquent, Iotapianus pourrait avoir appartenu à la famille royale de Commagène [45]. Pour certains auteurs modernes, cette référence à Alexandre le Grand impliquerait également un rattachement à Sévère Alexandre [46]. En tout état de cause, Jotapien était vraisemblablement un dignitaire local qui bénéficiait de nombreux soutiens au sein de I'aristocratie orientale. Nous savons peu de choses sur I'issue de ce coup d'État. Seuls Zosime et Aurelius Victor fournissent quelques informations sur ce sujet. Selon Zosime, Jotapien aurait été battu sans grande difficulté. Il ne nous donne pas de détails sur les circonstances et les auteurs de cette disparition. Aurelius Victor indique, quant à lui, qu'il fut assassiné par les soldats et sa tête apportée à Dèce [47]. L'absence de soutien militaire explique la rapide conclusion de son mouvement, quelques mois après son déclenchement, grâce à une probable intervention de Priscus [48].

S'agissant des attaques aux frontières, les sources ne font pas mention d'un regain de tension à la frontière orientale avec les Perses. L'accord de début de règne entre Philippe et Sapor, malgré les différentes d'interpré-

[43] KöRner 2002, p. 280 ; POTter 1990, p. 268-273.

[44] Aurelius Victor, Liber de Caesaribus, XXIX, 2.

[45] SyME 1971, p. 202 ; sur la dynastie des Commagènes, voir SulLivan 1977, p. 732-798; POTTER 1990, p. 248.

[46] BaRBIERI 1952, p. 405, 654 ; HaRTMANN 1982, p. 73 ; POTTER 1990, p. 248

[47] Aurelius Victor, Liber de Caesaribus, XXIX, 2.

[48] KÖRNER 2002, p. 280.

[49] PEACHIN 1991, p. 341.

[50] Bray 1997, p. 41 ; Christol 1975, p. 808.

[51] Valérien était issu de l'illustre famille sénatoriale des Licinii. Il aurait effectué une brillante carrière sénatoriale qui I'aurait amené à occuper plusieurs fonctions d'importance dont un consulat ordinaire en 238 et le gouvernement de tations évoquées plus haut, aurait garanti une certaine stabilité dans les provinces orientales sous la supervision de Priscus.

En définitive, le bilan du supra-commandement de Priscus semble positif. Pour ce qui est des attaques aux frontières, on observe une pause dans les incursions perses. Quant à la diminution des coups d'État, il est vrai que I'action de Priscus sur le plan fiscal fut à l'origine du coup d'État de Jotapien. Cependant, la rapide conclusion de cette insurrection démontre l'efficacité d'une coordination locale des actions militaires dans le but de faire face à ce type de mouvement. Par ailleurs, au-delà de l'aspect militaire, il faut souligner que l'action de Priscus contribua dans une certaine mesure à redynamiser le fonctionnement administratif des provinces orientales profondément déstabilisées par les attaques répétées des voisins perses [49].

La crise militaire aux frontières de l'empire avec la multiplication des fronts de combat rendait essentielle la mise en place des supra-commandements. Après les règnes de Trajan Dèce, Trébonien Galle et Émilien, c'est sous les règnes de Valérien et de Gallien que vont réapparaître la structure des supra-commandements, particulièrement à travers la position de Valérien le Jeune dans I'Illyricum et plus tard d'Odenath de Palmyre en Orient.

\section{GALLIEN ET LA CRÉATION DU SUPRA-COMMANDEMENT DANS L'ILLYRICUM}

Après son coup d'État contre Émilien en septembre 253 [50], I'empereur Valérien décide d'associer son fils Gallien au pouvoir. Ce choix stratégique de Valérien s'explique par son intime connaissance des enjeux politiques et militaires de l'époque et sa reconnaissance de l'impossibilité pour un seul empereur de faire face aux attaques des ennemis extérieurs de l'empire [51]. Ainsi, Gallien est d'abord élevé, en septembre 253, à la dignité de Nobilissimus Caesar [52] par le Sénat, avant d'être

plusieurs provinces. Selon I'auteur de I'Histoire Auguste, il aurait même reçu les ambassades africaines qui venaient annoncer la proclamation de Gordien Ier. Plus tard, sous le règne de Dèce, il eut la responsabilité des affaires intérieures pendant l'expédition de l'empereur contre les Goths. Sous le bref règne de Trébonien Galle, il fut également envoyé en Rhétie dans le but de réorganiser les troupes aux frontières en prévision d'éventuelles campagnes militaires contre les germains dans les provinces danubiennes. SHA, Vita Gordianorum, 9, 7-8; Aurelius Victor, Liber de Caesaribus, XXXII, 2.

[52] $A E$ 1967, 584 ; Aurelius Victor, Liber de Caesaribus, XXXII, 3 ; Eutrope, IX, 6 ; Epitome de Caesaribus, XXXII, 2 ; Pflaum 1966-1967, p. 175-182; CIL VIII 10132 et CIL VIII 10141 ; ZaCCARIA, 1976, p. 343-361; PEACHIN 1988, p. $219-224$ 
associé pleinement comme Auguste au début de 254 pour créer un système avec deux empereurs [53]. Ils disposaient tous les deux des mêmes pouvoirs, partageaient le Grand Pontificat et le titre de Père de la Patrie [54]. Toutefois, Valérien gardait une certaine préséance sur son fils [55]. Sur le plan administratif, deux secteurs d'action correspondant aux deux principaux foyers de tension étaient créés. Valérien s'occupait des provinces orientales et Gallien, des provinces occidentales [56]. À côté de ce partage de responsabilités entre les deux Augustes, Gallien décida d'effectuer un autre partage dans sa zone de responsabilité pour créer un supra-commandement dans I'Illyricum qu'il confia à son fils aîné, Valérien II ou Valérien le Jeune. Ainsi, il éleva dans la seconde moitié de 256 au titre de César, Publius Licinius Cornelius Valerianus [57]. Il était le fils de Gallien et de Cornelia Salonina [58]. Dans quel contexte fut donc créé ce supra-commandement dans l'Illyricum ? En effet, I'Illyricum qui correspondait aux Pannonies, Mésies et à la Dalmatie faisait I'objet d'attaques répétées de Sarmates et de Carpes depuis le règne de Philippe et Dèce [59]. Entre 254 et 255, Gallien y effectua une intense activité de redressement qui permit de rétablir une certaine sécurité aux frontières [60]. Ces succès militaires, qui furent I'objet de la célébration de la cinquième victoire impériale des Augustes [61], n'éloignaient pas pour autant les menaces d'attaques qui demeuraient persistantes. C'est donc pour consolider les victoires de 255 remportées sur les Germains que Gallien décida de créer en 256 un supracommandement qu'il confia à Valérien le Jeune. Cette structure participait à la politique dynastique initiée par Valérien et qui se manifestait entre autres par la présence d'un membre de la famille impériale dans chaque région sensible en vue d'assurer la coordination des actions militaires sur le théâtre des opérations. L'installation du jeune César en Illyricum doit se situer entre août et septembre 256 [62].

Sur le plan protocolaire, Valérien le Jeune occupait une position subordonnée vis-à-vis de son père et de son

[53] Christol 2006, p. 131.

[54] Ibid., p. 132 ; CIL XVI 155 ; ILS 2010.

[55] CIL XVI 155 ; ILS 2010 ; ChRISTOL 2006, p. 132 ; BRAY 1997 , p. 45

[56] Zosime, I, XXX, 1 ; BraY 1997, p. 45.

[57] PeACHIN 1990, p. 38 ; Kienast 1990, p. 217-218; De BloIs 1976, p. 24

[58] ZaCCARIA 1978, p. 59-155.

[59] Demougeot 1969, p. 437-438.

[60] Ibid., p. 439.

[61] Christol 1997, p. 244-245; RIC V 1, 141; RIC IV 1, 17-19.

[62] Christol 1997, p. 246. grand-père. Toutefois, il semble qu'à partir du début de I'année 258, ses pouvoirs se soient accrus [63] puisque son nom est mentionné aux côtés de ceux de son père et de son grand-père dans I'intitulé des constitutions impériales [64]. Nous ne sommes pas en mesure de clarifier la véritable nature des pouvoirs détenus par Valérien le Jeune ou encore ses relations avec les gouverneurs de province de l'Illyricum. À partir des milliaires découverts [65], nous pouvons supposer qu'il supervisait les opérations militaires avec une armée unique à sa disposition ainsi qu'un atelier monétaire particulier, celui de Viminacium [66]. Il avait au sein de son état-major des généraux expérimentés tels qu'Ingenuus et Régalien pour l'assister dans la coordination des opérations militaires sur le théâtre des opérations [67].

Quel fut l'impact de la création du supra-commandement dans I'Illyricum sur la diminution des coups d'État et l'arrêt des attaques germaniques dans cette région ?

Entre 258 et 259, la sécurité se détériora dans I'Illyricum à l'occasion notamment de la mort de Valérien le Jeune [68] et des coups d'État d'Ingenuus et de Régalien. Les circonstances réelles de la disparition de Valérien II nous sont inconnues. Il est probable, sur la base du témoignage d'Aurelius Victor, qu'il ait perdu la vie au cours d'une opération militaire contre les germains [69]. Par ailleurs, Pierre le Patrice fait état de la détérioration des rapports entre Ingenuus et Gallien du fait de la colère de l'impératrice Cornelia Salonina qui rendait Ingenuus responsable de la mort de son fils [70]. Cette colère de Cornelia suggère I'hypothèse que celui-là avait dans une certaine mesure failli à sa mission de protection du jeune César. Profitant du vide politique laissé par cette disparition et prétextant de la fermeture de l'atelier monétaire de Viminacium par Gallien, Ingenuus se fit acclamer empereur en 258 à Sirmium par les troupes basées en Pannonie inférieure [71]. Informé de cette situation, Gallien qui se trouvait en Gaule, alla affronter Ingenuus. Ce dernier fut défait au cours de la bataille décisive de Mursa, dans I'actuelle Croatie [72].

[63] Trois milliaires qui auraient été découverts dans la région de Vindobona attribuent le titre d'imperator à Valérien le Jeune. CIL III 4646, 4647, 4652.

[64] CJ 5, 3, $5=9$, 9, du 18 de mai 258.

[65] Pflaum 1966, p. 538- 542 ; Id., 1966-1967; Christol 1975, p. 816.

[66] FITZ 1966, p. 32-33.

[67] Demougeot 1969, p. 444.

[68] Aurelius Victor, Liber de Caesaribus, XXXIII, 2.

[69] Aurelius Victor, Liber de Caesaribus, XXXIII, 2.

[70] Pierre le Patrice, 192 ; DrinkWATER 1987, p. 103.

[71] Chastagnol, SHA, Triginta Tyranni, IX, 1.

[72] Aurelius Victor, Liber de Caesaribus, XXXIII, 2. 
Après le départ de Gallien de la région, un autre coup d'État fut déclenché par Régalien [73]. Cette insurrection serait à situer entre l'été et l'automne 259, précisément entre juillet et décembre 259 [74]. Selon I'auteur de I'Histoire Auguste, certains soldats, mécontents du rétablissement militaire effectué ar Gallien à l'issue de sa victoire sur Ingenuus, se seraient ralliés à Régalien pour initier cette insurrection.

« C'est ainsi que Regilianus qui exerçait un commandement militaire en Illyricum fut proclamé empereur ; il dut son élection aux Mésiens qui avaient été précédemment vaincus en même temps qu'Ingenuus et dont les familles avaient subi de cruels sévices de la part de Gallien »[75].

À la différence d'Ingenuus, Régalien put battre monnaie. Son monnayage se trouve essentiellement dans la région de Carnuntum en Pannonie supérieure [76]. On le retrouve aussi au sud de la Drave et en Pannonie inférieure [77]. Il aurait assumé, en remplacement d'Ingenuus, le gouvernement des provinces danubiennes de Pannonie et de Mésie [78]. Nous disposons de peu de sources d'informations sur les circonstances de la fin du mouvement de Régalien. Il est probable que son coup d'État se soit terminé après une attaque des Roxolans dans la région [79]. Qu'en est-il de l'impact de ce supracommandement dans la diminution des attaques aux frontières dans l'Illyricum ?

Censé consolider les conquêtes de Gallien entre 254 et 255 sur les Germains, le supra-commandement de I'Illyricum fut inefficace à cet égard. Valérien le Jeune fut rapidement tué au combat, ce qui ouvrit la porte aux coups d'État d'Ingenuus et de Régalien ainsi qu'à une guerre civile pour le rétablissement de l'autorité impériale. Il est évident que ces conflits internes affectèrent grandement la stratégie de protection des frontières face aux invasions. Les forces militaires prévues pour assurer la protection des frontières étaient désormais mobilisées pour rétablir l'autorité impériale face aux séditions, ce qui dans une certaine mesure favorisait les attaques germaniques dans la région.

Le supra-commandement dans I'Illyricum s'inscrivait dans la stratégie de partage de responsabilités entre les membres de la famille impériale. Il devait à terme favoriser une coordination efficace des opérations militaires dans l'empire en utilisant toutes les ressources d'une

[73] Aurelius Victor, Liber de Caesaribus, XXXIII, 2.

[74] FiTz 1966, p. 44.

[75] Chastagnol, SHA, Triginta Tyranni, X, 1.

[76] FITZ 1976, p. 37.

[77] Ibid., p. 47.

[78] Ibid., p. 44. politique dynastique. Toutefois, la jeunesse et l'inexpérience de Valérien le Jeune ne permit pas de contenir les ambitions de puissants et expérimentés généraux tels qu'Ingenuus et Régalien. Ces deux coups d'État renforcèrent la profonde instabilité de cette région. Les troupes romaines ne purent efficacement faire face aux assauts répétés des Alamans, Sarmates et Roxolans, qui avaient intensifié leurs attaques dans cette région en 257 [80].

\section{ODENATH DE PALMYRE ET LE SUPRA-COMMANDEMENT ORIENTAL}

Après le supra-commandement en Illyricum, c'est en Orient, avec la position d'Odenath, que I'on voit apparaître un autre supra-commandement, cette fois ci au cours du règne personnel de Gallien.

La capture de l'empereur Valérien par les Perses plongea les populations des provinces orientales dans un profond désarroi [81]. En effet, dans le partage de responsabilité de début de règne, Valérien coordonnait les activités militaires dans cette région. Il a d'ailleurs été à I'origine de plusieurs succès militaires contre les Perses [82]. Sa capture eut un impact négatif non seulement sur les opérations militaires en cours dans la région mais également sur le moral des populations provinciales. Il était important pour Gallien de consolider la présence romaine dans le but non seulement de rassurer les Orientaux mais également de contenir l'expansion perse. Occupé par la pacification des provinces occidentales en proie aux attaques germaniques, Gallien n'avait d'autre choix que de renforcer la position politique d'Odenath de Palmyre en créant de facto un supra-commandement oriental. De plus, après la disparition de ses deux fils, Gallien ne disposait plus de proches pour continuer la politique dynastique initiée par son père. Il est important dans I'analyse de ce supra-commandement de bien comprendre la position d'Odenath dans le contexte politique de I'Orient romain au III siècle.

L'onomastique officielle d'Odenath était Septimius Odenathus. Il serait né au début du III ${ }^{\mathrm{e}}$ siècle [83]. Ses ascendants faisaient partie de ceux qui avaient reçu la citoyenneté romaine sous Septime Sévère. Sa famille devrait donc être issue de la noblesse palmyrénienne

[79] FITz 1966, p. $50 ; 61-63$

[80] Demougeot 1969, p. 446.

[81] Zosime, I, 37, 1.

[82] Christol 2006, p. 133 ; RIC V 1, Valérien (Parthicus Maximus).

[83] Michel Gawlikowski propose 220 comme date de sa naissance, voir StARCKY \& GAWLIKOWSKI 1985, p. 261. 
depuis plusieurs générations [84]. Il a longtemps existé une confusion sur les origines d'Odenath. En effet, le continuateur anonyme de Dion Cassius avait fait référence à deux Odenath, un ancien et un jeune. Cette information avait amené à croire qu'Odenath aurait été le fils d'un autre Odenath nommé Odenath I'Ancien. Aujourd'hui, grâce à une inscription publiée par Michel Gawlikowski, on est en mesure d'affirmer que Septimius Odenath était le fils de Septimius Hairan qui, lui-même, était le fils de Waballath [85]. Odenath eut trois fils pour lesquels nous avons des références épigraphiques et littéraires [86]. Il s'agit d'Hérodien et d'Hairan issus d'un premier mariage, et de Septimius Waballathus, le fils de sa seconde épouse Zénobie. Sous le règne de Philippe, il aurait été promu sénateur [87].

À partir de 252, Sapor renouvelle ses attaques en territoire romain, plaçant ainsi la cité de Palmyre dans une situation extrêmement délicate, puisqu'elle se trouvait à la frontière des deux empires [88]. Pour pallier l'absence de soutien énergique du pouvoir impérial romain sous Trébonien Galle, Odenath se serait vu confier par la population locale la charge d'exarque de Palmyre [89]. Cette fonction devait probablement comporter un important pan militaire, compte tenu du contexte dans lequel elle fut attribuée à Odenath. Udo Hartmann confirme I'attribution de cette magistrature par les Palmyréniens et non par l'empereur romain [90]. Odenath associa son fils Hairan à cette magistrature exceptionnelle en créant ainsi une structure héréditaire [91]. Entre 257 et 258 , sous le règne conjoint des empereurs Valérien et Gallien, Odenath revêtit les titres de Vir Consularis et de Legatus Augusti Propraetore provinciae Syriae Phonices [92]. L'octroi de ces titres à Odenath fait partie de la pratique bien connue des empereurs de conférer des ornementa à certains personnages éminents. En effet, les ornementa sont des décorations attribuant à vie à un personnage des signes extérieurs d'une magistrature qu'il a ou non déjà obtenue [93]. Dans le cas d'Odenath, l'octroi de ses titres impliquait qu'il était considéré comme ancien consul et à ce titre, gouverneur de la province impériale de Syrie Phénicie dans laquelle était incluse Palmyre. C'est ce que confirme une inscrip- tion élevée par la corporation des orfèvres de Palmyre à Odenath :

« Septimius Odainat, l'illustre consulaire, notre seigneur, élevé par la corporation des orfèvres... » [94].

Il est probable qu'il ait reçu tous ces honneurs de Valérien qui se trouvait en Orient à cette période.

En 260, les troupes romaines furent défaites et I'empereur Valérien fut fait prisonnier par les Perses. Toutefois, sur leur chemin de retour, ceux-ci furent stoppés par Odenath. Plus tard, Odenath contribua à mettre fin au coup d'État de Macrien et de ses fils en Orient. En récompense de son rôle stratégique dans la lutte contre l'ennemi perse mais également dans le maintien de la sécurité dans les provinces orientales de l'Empire, Odenath se vit offrir par Gallien le supra-commandement oriental que I'on reconnaît dans les titres de dux Romanorum [95] puis de corrector totius Orientis [96]. C'est ce que souligne Zonaras :

«Gallien envoya cependant Odenat chef des Palmyréniens, contre Quintus fils puîné de Macrin qui s'était emparé de presque tout l'Orient. Mais la nouvelle de la défaite de Macrin et de Macrien n'eut pas sitôt été répandue que plusieurs villes secouèrent le joug de I'obéissance de Quintus et de Balliste. Odenat les attaqua près d'Émèse, les vainquit, tua Balliste, et à son exemple, les habitants tuèrent Quintus. L'Empereur récompensa la valeur et les services d'Odenat du commandement des troupes d'Orient, où il acquit beaucoup de gloire en combattant diverses nations, et même les Perses » [97].

Il importe à ce stade de clarifier les deux titres attribués à Odenath. D'abord celui de dux Romanorum. Au III siècle, le titre de dux était donné à un membre de l'ordre équestre. Les attributions des duces étaient d'ordre à la fois militaire et administratif [98]. Cette montée en puissance des duces, au milieu du III siècle, trouve son origine dans le rôle croissant joué par les chevaliers dans I'appareil de I'État depuis le milieu du II siècle [99]. La réforme administrative de Gallien vient consacrer à cet égard un état de fait [100]. Ce titre démontre clairement le commandement militaire d'Odenath sur les troupes romaines et palmyréniennes en Orient. Cependant, il nous est impossible d'indiquer jusqu'où, sur le plan
[84] HaRTMANN 2001, p. 88.

[85] STARCKY \& GAWLIKOWSKI 1985, p. 257.

[86] Ibid., p. 252-253.

[87] Ibid., p. 261.

[88] PotTer 1990, p. 291-293; EdWeLl 2007, p. 185.

[89] HARTMANN 2001, p. 92-93.

[90] Ibid., p. 97.

[91] STARCKY \& GAWLIKOWSKI 1985, p. 58.

[92] Le BOHEC 2009, p. 204.
[93] BenOIST 2000, p. 311, 320-321.

[94] StaRCKy \& GaWLiKowski 1985, p. 58.

[95] Zonaras, XII, 23.

[96] PotTer 1996, p. 272.

[97] Zonaras, XII, 24.

[98] SOUTHERN 2001, p. 439.

[99] CHRISTOL 1986, p. 46.

[100] Ibid., p. 39-44. 
géographique, s'étendait cette autorité et si elle incluait des attributions administratives. S'agissant du titre de corrector, il faut indiquer que, sous l'empire, il était donné à des officiers sénatoriaux chargés par l'empereur de redresser les finances publiques de certaines citées orientales. Ces personnages étaient détenteurs de l'imperium et étaient indépendants des gouverneurs de provinces [101]. Ce statut de corrector d'Odenath est confirmé par une inscription en langue palmyrénienne datée de 271 [102]. Il s'agit d'une inscription posthume accompagnant une statue érigée en I'honneur d'Odenath par le général palmyrénien Zabdas. À cette première inscription, on ajoute pour les besoins de l'analyse le texte d'un milliaire érigé en I'honneur de Waballath. Sur la première, Odenath est commémoré en tant que $m / k m / k^{\prime} w m t q n n$ dy $m d n h^{\prime} k l h$, ce qui correspond à Roi des rois et corrector totius Orientis [103]. Sur le second, Waballah est présenté comme $m / k m / k^{\prime} w^{\prime}$ pnrtht ${ }^{\prime} d y m d n h^{\prime} k l h$ [104] ce qui correspond également au titre de Roi des rois avec I'ajout du terme pnrtht' qui serait une variante du terme mtqnn'. L'utilisation de deux différents termes pour traduire le titre de corrector du terme palmyrénien pnrtht' dans I'inscription honorifique à Waballah, a donné lieu à de vifs débats entre les spécialistes [105]. Certains arguant que cela suggérait qu'Odenath avait une position différente de son fils et que la traduction du terme palmyrénien $m d n h^{\prime}$ par restitutor serait préférable à celle de corrector puisqu'elle n'impliquait pas une position formelle dans la structure administrative romaine [106]. Au-delà de la technicité des titres d'Odenath, il est indéniable que celuici avait obtenu de Gallien une large autorité sur Palmyre mais aussi sur l'ensemble des provinces orientales [107]. Nous ne sommes malheureusement pas en mesure de confirmer la nature réelle de cette autorité. Nous pensons qu'il faudrait en conséquence la rapprocher de celle accordée à Priscus par Philippe. Cette fonction n'avait pas de contenu en matière de droit public mais sur le plan politique et militaire elle accordait à Odenath une autorité de supervision sur l'Orient romain [108]. Il importe de préciser que les gouverneurs des provinces continuaient d'être nommés par Gallien, ce qui confirme qu'Odenath n'avait pas de commandement direct sur ces provinces [109]. En tout état de cause, l'absence de sources d'information

[101] JESSEN 1913, s. v. Helios, RE IV, 8, col. 1645-1655; JacQues \& Scheid 2010, p. 269 ; LefebVRe 2011, p. 133-134. [102] Dodgeon \& LieU 1991, p. 88 notes 4, 7, 2.

[103] CIS II 3946 ; SWAIN 1993, p. 157 ; POTTER 1996, p. 272.

[104] CIS II 3971 ; MASSON 1974, p. 442.

[105] SWAin 1993, p. 157 ; POTTER 1996, p. 272.

[106] SWAIN 1993, p. 159-160.

[107] POTTER 1990, p. 390-394.

[108] POTTER 1996, p. 272. empêche de clarifier la nature et l'étendue de cette autorité attribuée à Odenath par Gallien.

Le symbolisme politique de cette nouvelle fonction attribuée à Odenath est fondamental puisqu'il permet de comprendre la distribution des pouvoirs dans l'empire à cette période. En effet, avec la capture de Valérien, il était important pour le pouvoir central romain de combler le vide politique à travers la présence d'une forte autorité dans la région pour faire face aux assauts perses. La nouvelle position d'Odenath grâce à ce supra-commandement se comprend donc dans le contexte politique de maintien de l'équilibre des forces dans une région hautement stratégique pour l'empire. Quelles furent les conséquences de ce supra-commandement octroyé à Odenath dans le rétablissement de la paix en Orient?

Pour ce qui est des coups d'État, il faut souligner qu'Odenath joua un rôle décisif dans la répression de la révolte de Macrien et de ses fils. En effet, Macrien, qui était le plus gradé des généraux de Valérien, décida avec I'appui du préfet du prétoire Balliste de combler le vide institutionnel occasionné par la capture de Valérien en faisant acclamer empereurs ses fils : Quietus et Macrien le Jeune [110]. Ceux-ci furent reconnus dans plusieurs régions orientales, notamment en Syrie, en Cappadoce et surtout en Égypte [111]. Des documents d'archives égyptiens indiquent que la première année de règne des deux empereurs serait à situer autour du 29 août 260 [112]. Macrien et son fils Macrien le Jeune, qui tentaient de conquérir certaines provinces occidentales, furent défaits par Aureolus dans I'Illyricum, ce dernier agissant au nom de l'empereur Gallien [113].

Odenath quant à lui marcha contre Balliste et Quietus sur instruction de Gallien [114] et les assiégea dans Émèse, capitale de la province de Syrie, où ils furent massacrés par la population locale [115]. L'action d'Odenath fut déterminante pour mettre fin au coup d'État de Macrien. En ce qui concerne les invasions, Odenath fut particulièrement actif dans la protection des provinces orientales contre les Perses particulièrement après la capture de Valérien. En effet, il mena deux importantes campagnes contre les Perses entre 262 et 268 [116].

[109] Pflaum 1953, p. 307-330.

[110] Chastagnol, SHA, Triginta Tyranni, XII, 1-4.

[111] CHRISTOL 2006, p. 144.

[112] Christol 1975, p. 818.

[113] Chastagnol, SHA, Triginta Tyranni, XII, 13 ; Zonaras, XII, 24.

[114] Zonaras, XII, 24 ; Zosime, I, 39, 1.

[115] Zonaras, XII, 24.

[116] Le BOHEC 2009, p. 205-206. 
La première, qui se déroula entre 262 et 263, eut un remarquable succès [117]. Le corps expéditionnaire était composé de soldats romains et d'archers palmyréniens. Odenath libéra plusieurs cités sous domination iranienne dont Carrhae et put reprendre le contrôle de la Mésopotamie. Il atteignit même Ctésiphon, la capitale de l'empire perse [118]. Cette brillante campagne d'Odenath permit à Gallien de revêtir le titre de Persicus Maximus en 263 [119].

La seconde campagne d'Odenath fut menée autour de 267 [120]. Au cours de cette deuxième campagne, les troupes palmyréniennes remportèrent d'importants succès et se dirigeaient encore vers la capitale perse lorsqu'Odenath fut assassiné avec son fils Hérodien, entre le 30 août 267 et le 29 avril 268 [121].

Le supra-commandement oriental d'Odenath s'inscrit dans la continuité de celui octroyé à Priscus sous Philippe I'Arabe. La présence de l'ennemi perse rendait nécessaire la consolidation de l'autorité romaine dans la région. De plus, il venait combler le vide laissé par la capture de l'empereur Valérien. Les compétences stratégiques et tactiques d'Odenath permirent de garantir la sécurité tout en maintenant une certaine stabilité politique dans I'Orient romain. Toutefois, I'octroi d'une si grande autorité aux contours imprécis à un personnage extérieur à la famille impériale prépara la sécession palmyrénienne sous Waballath et Zénobie.

\section{CONCLUSION}

Trois conclusions sont à tirer de cette analyse des supracommandements sous les règnes de Philippe I'Arabe et Gallien. La première concerne le contexte de leur mise en place. L'instauration des supra-commandements participe de la stratégie de stabilisation des frontières orientale et occidentale grâce à l'établissement de structures décentralisées de coordination des opérations militaires. Ainsi, Valérien le Jeune fut installé dans I'Illyricum pour non seulement raffermir les conquêtes militaires de 255 mais également pour prévenir d'éventuelles attaques des Germains dans la région. Dans la même perspective, Priscus puis Odenath reçurent successivement le supracommandement oriental pour contenir les attaques perses en territoire romain.

La deuxième conclusion relève de la nature des supracommandements et de l'autorité de leurs détenteurs. Sur la base des sources d'informations disponibles nous pouvons déduire que ces structures n'avaient pas un contenu administratif défini en matière de droit public. Toutefois, elles conféraient à leur détenteur une préséance politique sur les autres gouverneurs de provinces et une autorité de supervision des opérations militaires dans la région concernée. Ils avaient pour mandat de superviser la stratégie de lutte contre les attaques des ennemis extérieurs aux frontières ; d'où leur création au sein des zones menacées d'attaques.

La troisième conclusion touche l'identité des détenteurs de ces supra-commandements. Pour la période analysée, exception faite d'Odenath de Palmyre, ils étaient issus de la famille impériale. Ce choix d'un membre de la famille impériale garantissait la fidélité du détenteur à l'empereur et limitait dans le même temps les risques d'un coup d'État.

En définitive, il faut souligner que les supra-commandements doivent être perçus comme une réponse des empereurs du III ${ }^{\mathrm{e}}$ siècle à la multiplicité des foyers de tension et à leur impossible présence physique sur tous les théâtres d'opérations. Par ailleurs, ils démontrent, dans une certaine mesure, leur capacité à adapter les structures administratives héritées du compromis augustéen aux nouvelles exigences politiques et militaires du moment. Ils préfiguraient à cet égard, les transformations politiques et administratives de l'époque de Dioclétien.

[117] HARTMANN 2001, p. 162-164.

[118] Zosime, I, 39, 1-2; CHASTAGNOL, SHA, Triginta Tyranni, XV, 3.

[119] CIL VIII 22765 ; ILS 8923 ; HaRTMANn 2001, p. 68.

[120] Le Bohec 2009, p. 206 ; StARCKy \& GaWLikowski 1985, p. 60 .

[120] StaRcky \& GaWLikowski 1985, p. 62 


\section{SOURCES}

Sextus Aurelius Victor, Le Livre des Césars, texte traduit et commenté par Pierre Dufraigne, Les Belles Lettres, Paris, 1975. Pseudo Aurelius Victor, Abrégé des Césars, texte établi et commenté par Michel Festy, Les Belles Lettres, Paris, 1999. Histoire Auguste, texte traduit et commenté par André Chastagnol, Paris, 1994.

Jean Zonaras, The History of Zonaras: From Alexander Severus to the Death of Theodosius the Great, traduit par Thomas Banchich \& Eugene Lane, London, 2009.

Zosime, Histoire Nouvelle, tome 1, livres I-II, texte traduit et établi par François Paschoud, Les Belles Lettres, Paris, 2000.

Badel, Christophe \& Bérenger, Agnès, 1998, L'Empire romain au III siècle après J.-C. (Regards sur l'histoire. Histoire ancienne), Paris.

BarberI, Guido, 1952, L'albo senatorio da Settimio Severo a Carino. 193-285, Roma.

Benoist, Stéphane, 2000, « Le prince et la société romaine d'Empire au III siècle : le cas des ornamenta 》, CCGG 11, p. 309329.

Bérenger, Agnès, 2004, « Le contrôle des gouverneurs de province sous le Haut-Empire », Contrôler les agents du pouvoir, Limoges, p. 127-146.

BraY, John, 1997, Gallienus: a study in reformist and sexual politics, Kent Town.

Chaumont, Marie-Louise, 1969, Recherches sur l'histoire d'Arménie, de l'avènement des Sassanides à la conversion du royaume, Paris.

Christol, Michel, 1975, «Les règnes de Valérien et Gallien (253-268) : Travaux d'ensemble, questions chronologiques », ANRW, II, 2, Berlin - New York, p. 803-827.

Christol, Michel, 1976, «Une carrière équestre sous le règne de Gallien », Latomus 35, p. 866-874.

Christol, Michel, 1997, «Les déplacements du collège impérial de 256 à 258 : Cologne, capitale impériale », CCGG 8, p. $243-253$.

Christol, Michel, 2006, L'Empire romain du III siècle : Histoire Politique (1 $1^{\text {re }}$ éd. 1997), Paris.

Christol, Michel \& Bourgeors, Ariane, 1973, « À propos d'un antoninianus inédit de Valérien le Jeune provenant du trésor de Chézelles (Indre) : la date d'ouverture de l'atelier de Cologne », Bulletin de la Société française de Numismatique 108, p. 415-418.

DE BloIs, Lukas, 1978-1979, « The reign of the emperor Philip the Arabian », Talanta 10-11, p. 11-43.

Demougeot, Emilienne, 1969, La formation de l'Europe et les invasions barbares, 1. Des origines germaniques à l'avènement de Dioclétien, Paris (Collection historique).

Dodgeon, Michael H. \& Lieu, Samuel N. C, 1991, The Roman Eastern Frontier and the Persian Wars (AD 226-363). A Documentary History, London.

Drinkwater, John, 1987, The Gallic Empire. Separatism and Continuity in the North Western Provinces of the Roman Empire A.D. 260-274, Wiesbaden - Stuttgart.

Edwell, Peter, 2008, Between Rome and Persia. The Middle Euphrates, Mesopotamia and Palmyra under Roman control, London.

Feissel, Denis \& Gascou, Jean, 1995, « Documents d'archives romains inédits du Moyen-Euphrate (III S. après J-C) », Journal des savants 1 (1), p. 65-119.

Fitz, Jenö, 1965, « Tullius Menophilus », Acta Anta. Acad. 13, p. 433-440.

Fitz, Jenö, 1966, Ingenuus et Régalien, Bruxelles (Collection Latomus 81).

HaRtman, Felix, 1982, Herrscherwechsel und Reichskrise: Untersuchungen zu den Ursachen und Konsequenzen der Herrscherwechsel im Imperium Romanum der Soldatenkaiserzeit (3. Jahrhundert n. Chr.), Frankfurt-am-Main.

HaRtmann, Udo, 2001, Das Palmyrenische Teilreich, Stuttgart.

Howe, Laurence Lee, 1942, The Pretorian Prefect from Commodus to Diocletian (A.D. 180-304), Chicago.

JACQues, François \& SCHeId, John, 2000, Rome et l'intégration de l'Empire, 1. Les structures de l'Empire (1 ${ }^{\text {re }}$ éd. 1990), Paris (Nouvelle Clio).

Kettenhofen, Erich, 1982, Die römisch-persischen Kriege des 3. Jahrhunderts $n$. Chr.: nach der Inschrift Sähpuhrs I. an der Ka'be-ye Zartost' (SKZ), Wiesbaden.

KIENAST, Dietmar, 1990, Römische Kaisertabelle: Grundzüge einer römischen Kaiserchronologie, Darmstadt.

Körner, Christian, 2002, Philippus Arabs. Ein Soldatenkaiser in der Tradition des antoninisch severischen Prinzipats, Berlin.

Le Bohec, Yann, 2009, L'armée romaine dans la tourmente. Une nouvelle approche de la «crise du siècle », Paris - Monaco.

Lefebvre, Sabine, 2011, L'administration de l'Empire romain : d'Auguste à Dioclétien, Paris. 
LoRiot, Xavier, 1975a, « Les premières années de la grande crise du III ${ }^{\text {e }}$ siècle. De l'avènement de Maximien de Thrace (235) à la mort de Gordien III (244) », ANRW, II, 2, Berlin - New York, p. 657-787.

Loriot, Xavier, 1975b, «Chronologie du règne de Philippe l'Arabe (244-249 après J-C.) », ANRW, II, 2, Berlin - New York, p. 788-797.

LoRIot, Xavier, 1994, «Quelques antoniniens de Pacatien trouvés en Gaule », BSFN 49, p. 844-848.

Loriot, Xavier \& Nony, Daniel, 1997, La crise de l'empire romain (235-285), Paris (Collection U 225).

MAson, Hugh J., 1974, Greek terms for Roman institutions: A Lexicon and Analysis, Toronto (American studies of papyrology 13).

MaricQ, André, 1958, «Res Gestae Divi Saporis », Syria 35, p. 295-360.

Millar, Fergus, 1993, The Roman Near East 31 B.C.-337 A.D, Cambridge, MA.

NASTI, Fara, 1993, «Un nuovo documento dalla Siria sulle competenze di governatori e procuratori in tema di interdetti », Index 21, p. 365-380.

NASTI, Fara, 1997, «Il prefetto del pretorio di CIL VI 1638 (= D. 1331) e la sua carriera », ZPE 117, p. 281-290.

Peachin, Michael, 1988, «Gallienus Caesar? », ZPE 74, p. 219-224.

Peachin, Michael, 1990, Roman imperial titulature and chronology, A.D. 235-284, Amsterdam.

Peachin, Michael 1991, «Philip's Progress: From Mesopotamia to Rome in A.D. 244 », Historia 40 (3), p. $331-342$.

Pekàry, Thomas, 1961, « Le «tribut » aux Perses et les finances de Philippe l'Arabe », Syria 38, p. $275-283$.

Pflaum, Hans-Georg, 1960, Les carrières procuratoriennes équestres sous le Haut-Empire romain, Paris, 4 vol.

Prlaum, Hans-Georg, 1966-1967, « P. Licinius Gallienus nobilissimus Caesar et Imp. M. Aurelius Numerianus nobilissimus Caesar Aug. à la lumière de deux nouveaux milliaires d'Oum el Bouaghi », Bull. Archéol. Algérienne 2, p. 175-182.

Potter, David, 1990, Prophecy and History in the Crisis of the Roman Empire: A Historical Commentary on the Thirteenth Sibylline Oracle, London.

Potter, David, 1996, « Palmyra and Rome: Odaenathus' Titulature and the Use of the Imperium Maius. », ZPE 113, p. 271-285.

Potter, David, 2004, The Roman Empire at Bay: AD 180-395, London - New York (Routledge history of the ancient world).

Rey-CoquaIs, Jean-Pierre, 1978, « Syrie romaine, de Pompée à Dioclétien », JRS 68, p. 44-73.

Southern, Pat, 2008, Empress Zenobia: Palmyra's Rebel Queen, London.

Starcky, Jean \& Gawlikowski, Michel, 1985, Palmyre, Paris.

Sullivan, Danielle, 1977, «The dynasty of Commagene», ANRW, II, 8, p. 732-798.

Syme, Ronald, 1987, « Avidius Cassius: His rank, Age and Quality », dans Johannes Straub et al. (éd.), Bonner Historia Augusta Colloquium 1984/1985, Bonn (Antiquitas, Reihe 4, Beiträge zur Historia Augusta Forschung 19), p. $207-222$.

SwaIN, Simon, 1993, " "Greek into Palmyrene: Odaenathus as 'Corrector Totius Orientis'? », ZPE 99, p.157-164.

Trout, Dennis E., 1989, « Victoria Redux and the First Year of the Reign of Philip the Arab » Chiron 19, p. 221-233.

VervaEt, Frederik, 2007, «The Reappearance of the Supra-Provincial Commands in the Late Second and Early Third Centuries C.E.: Constitutional and Historical Considerations », dans Olivier Hekster, Gerda de Kleijn \& Daniëlle Slootjes (éd.), Crises and the Roman Empire: Proceedings of the Seventh Workshop of the International Network Impact of Empire (Nijmegen, June 20-24, 2006), Leiden - Boston, p. 125-140. 